Supporting Information

\title{
Trackable water-soluble prodrug micelles capable of rapid mitochondrial-targeting and alkaline pH-responsive drug release for highly improved anticancer efficacy
}

Bei-Yu Liu, Xian-Ling Yang, Xiu Xing, Jun Li, Yan-Hong Liu, Na Wang* and Xiao-Qi Yu*

Key Laboratory of Green Chemistry \& Technology, Ministry of Education, College of Chemistry, Sichuan University, Chengdu 610064, P. R. China

\section{Contents}

1. Materials and Measurements .............................. S2

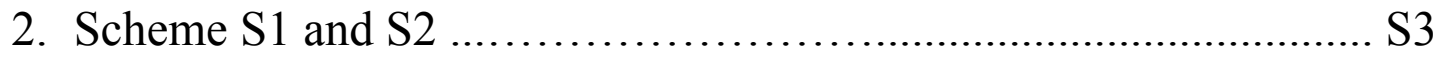

3. The preparation of DOX@(TPP)Cb-NA-PEG-NA-Cb(TPP)...... S3

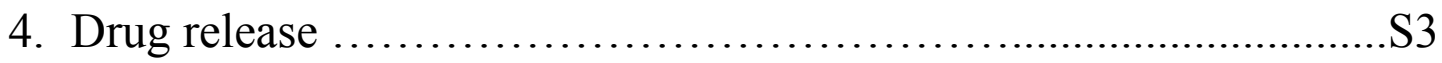

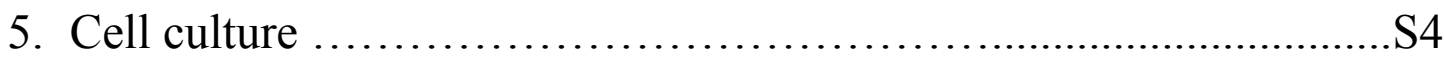

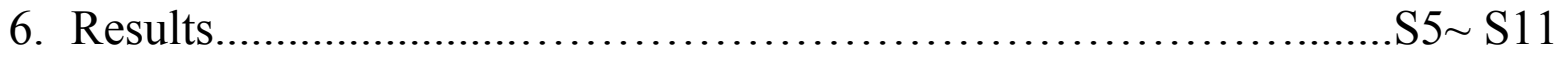

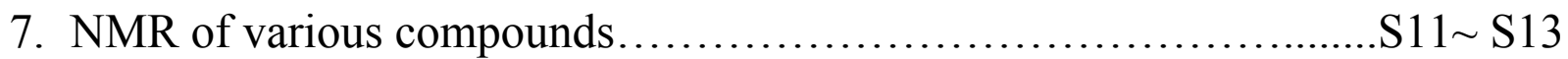

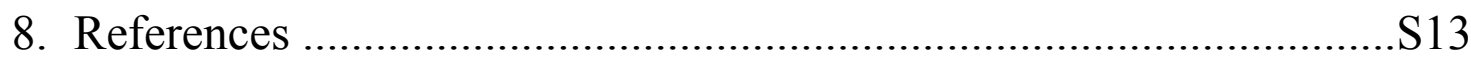

* Correspondence to: Fax./ Tel: +862885415886

N. Wang (E-mail: wnchem@scu.edu.cn), X.-Q. Yu (E-mail: xqyu@scu.edu.cn) 


\section{Materials and Measurement}

MTS 3-(4,5-dimethylthiazol-2-yl)-5-(3-carboxymethoxy-phenyl)-2-(4-sulfophenyl)-2H-tetrazolium was purchased from Sigma-Aldrich (St. Louis, MO), PEG, CuBr (99\%), and $N, N^{\prime}, N^{\prime}, N^{\prime \prime}, N^{\prime \prime}-$ pentamethyldietylenetriamine (PMDETA, 99\%) were purchased from Aladdin reagent (Shanghai, China), chlorambucil (98\%) and 4-piperidineethanol (96\%) was purchased form J \& K Scientific respectively, and used as received. Dichloromethane (DCM) and Dimethylformamide (DMF), tetrahydrofuran (THF) were dried and distilled first with purification. Ultra-pure water was used in the experiments. All other agents and solvents were purchased from commercial sources and used directly without further purification.

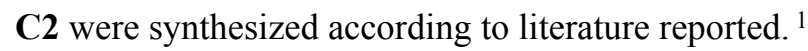

${ }^{1} \mathrm{H}$ NMR spectra were obtained on a Bruker DMX400 Spectrometer in $\mathbf{C D C l}_{\mathbf{3}}$ and given in relative to internals reference TMS standard. HRMS spectral data were measured on a Bruker Daltonics Bio TOF mass spectrometer. Gel permeation chromatography (GPC) to confirm the polymer molecular weights and their distributions was carried out on a Waters HPLC system equipped with a model 1515 isocratic pump, a 717 plus autosampler, and a 2424 refractive index (RI) detector with Waters Styragel ${ }^{\circledR}$ HT3 and HT4 columns in series. The eluting solvent was $\mathbf{T H F}$ at a flow rate of $1.0 \mathrm{~mL} / \mathrm{min}$ at $45^{\circ} \mathrm{C}$. The retention times were calibrated against poly (ethylene glycol) standard with the molecular weight range $600-80000$ Da. Transmission electron microscopy (TEM, Hitachi H-600) at an acceleration voltage of $100 \mathrm{kV}$ was performed to investigate the micelle morphology. The samples were prepared by dropping micellar solution onto a copper grid followed by negatively staining with a $1 \mathrm{wt} \%$ aqueous solution of phosphotungstic acid, respectively. The size of the micelles was determined using dynamic light scattering (DLS) with the micellar solutions filtered through a $0.45 \mu \mathrm{m}$ syringe filter prior to measurement. The measurements were carried out at $25{ }^{\circ} \mathrm{C}$ using a Zetasizer Nano-ZS90 system from Malvern Instruments equipped with a $633 \mathrm{~nm}$ He-Ne laser using backscattering detection with a fixed detector angle of $90^{\circ}$. The critical micelle concentration (CMC) was determined via fluorescence spectrometer. Fluorescence emission spectra were recorded on a F-7000 FL Spectrofluorophotometer (HITACHI) at $298 \mathrm{~K}$. Absorption spectra were recorded on a PERSEE TU-1901 UV-Visible Spectrophotometer. MTS method was used for testing the cell viability instead of MTT. Cells were obtained from Shanghai Institute of Biochemistry and Cell Biochemistry and Cell Biology, Chinese Academy of Science. 
2. Followed by the similar facile synthesis we combined as before ${ }^{2}$.

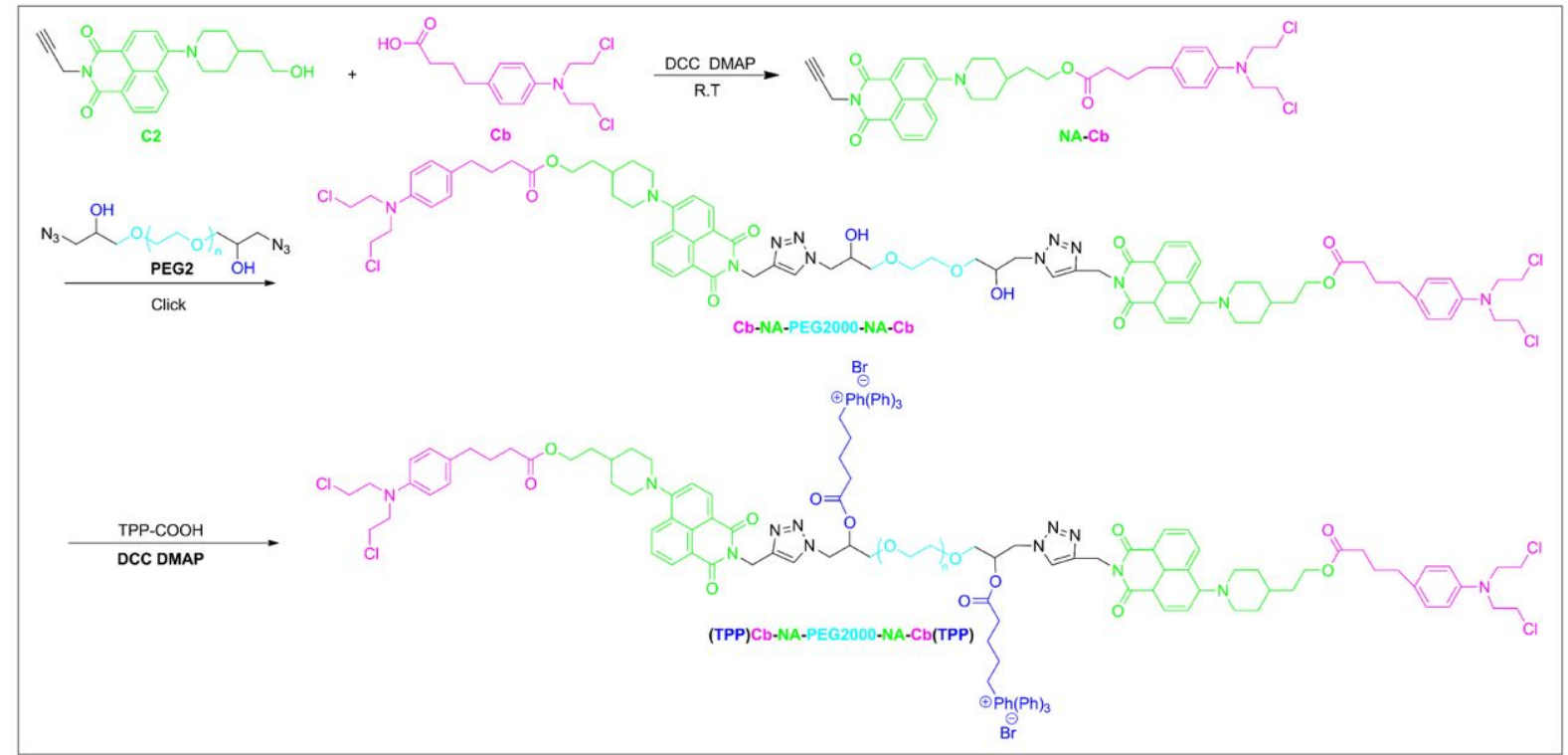

Scheme S1 The route of the synthesis of amphiphilic prodrugs.

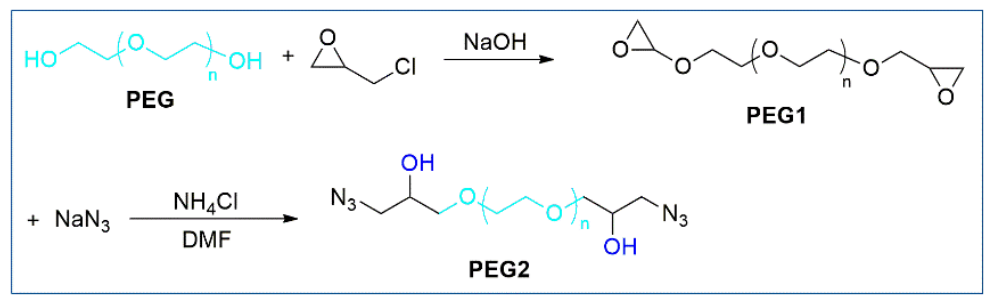

Scheme S2 Convenient synthesis of heterobifunctional clickable PEG with narrow polydispersity for the further modification

\section{The preparation of DOX@(TPP)Cb-NA-PEG2000-NA-Cb(TPP)}

Encapsulation of DOX was prepared by a film hydration method according to the study we reported before. ${ }^{3}$ And the $\mathbf{L E}$ and $\mathbf{E E}$ were tested after lyophilization via $\mathbf{U V}$-Vis analysis.

\section{Drug release}

The DOX@(TPP)Cb-NA-PEG2000-NA-Cb(TPP) micelles solution which was transferred to a dialysis tubing were incubated in vitro simulation biological environment in phosphate buffer saline (PBS, $10 \mathrm{mM}$, $0.5 \%$ Tuween-80) upon three different $\mathrm{pH}$ at $37{ }^{\circ} \mathrm{C}$, and samples at different time intervals which were totally replaced by fresh PBS were carried out to be tested by $\mathbf{U V}$-vis spectra or fluorescence spectral analysis against free Cb or DOX standard (Fig. S10 or Fig. S9). 


\section{Cell culture were carried out following previously reported procedures. ${ }^{1}$}

\subsection{Celluar internalization}

5.1.1 Cell imaging and mitochondrial target HeLa cells were pre-washed twice and then incubated with 50 $\mu \mathrm{g} \mathrm{mL}^{-1}$ prodrugs and MT DeepRed (commercially available mitochondrial dye, Mito Tracker Deep Red FM) at $37^{\circ} \mathrm{C}$ for separately $0.5 \mathrm{~h}$ or $1 \mathrm{~h}$ and 15 minutes. Then the cells were washed to remove unbounded probes before in situ imaging by confocal laser scanning microscopy analysis (CLSM, ZEISS LSM 780) at excitation wavelengths of $405 \mathrm{~nm}$ for NA (blue channel) and $633 \mathrm{~nm}$ for MT DeepRed (red channel), respectively. For DOX@(TPP)Cb-NA-PEG2000-NA-Cb(TPP), we added green channel at excitation wavelengths of $488 \mathrm{~nm}$ for DOX. For free DOX, there were green channel and red channel at excitation wavelengths of $488 \mathrm{~nm}$ for DOX and $633 \mathrm{~nm}$ for MT DeepRed, respectively.

5.1.2 Cell imaging and endolysosomal colocalization HeLa cells were pre-washed twice and then incubated with $50 \mu \mathrm{g} \mathrm{mL}-1$ prodrugs and LysoTracker Red at $37^{\circ} \mathrm{C}$ for separately $0.5 \mathrm{~h}$ or $1 \mathrm{~h}$ and 15 minutes. Then the cells were washed to remove unbounded probes before in situ imaging by confocal laser scanning microscopy analysis (CLSM, ZEISS LSM 780) at excitation wavelengths of $405 \mathrm{~nm}$ for NA (blue channel) and $546 \mathrm{~nm}$ for LysoTracker Red (red channel), respectively.

5.1.3 Colocalizaton ratio analysis was calculated based on the results from 5.1.1 and 5.1.2, such as Fig. 1(d) and Fig. 2(b). ${ }^{4}$

5.2 Mechanisms of cellular internalization To verify the possibility of quick mitochondrial targeting ability, the internalization mechanism of targeted prodrugs were studied following the same treatment according to other research our group reported. ${ }^{5}$ And the results were carried out from average intensity ratios from their CLSM images. ${ }^{6}$

5.3. Cytotoxicity assay Toxicity was determined by MTS method. About 10000 cells per well were seeded in $96-$-well plates and cultured overnight for $70-80 \%$ cell confluence. The medium was replaced with $100 \mu \mathrm{L}$ of fresh medium with $10 \% \mathrm{FBS}$, to which also with different tested concentrations of prodrugs. 48 hours later, $20 \%$ MTS solution in PBS was added to each well for additional incubation. The absorbance was measured using an ELISA plate reader (model 680, BioRad) at a wavelength of $490 \mathrm{~nm}$. The metabolic activity of the 
prodrugs treated cells was expressed as a relative to untreated cell controls taken as $100 \%$ metabolic activity. The concentrations of prodrugs were calculated by their theoretical value of molecular weights of prodrugs.

\section{Results}

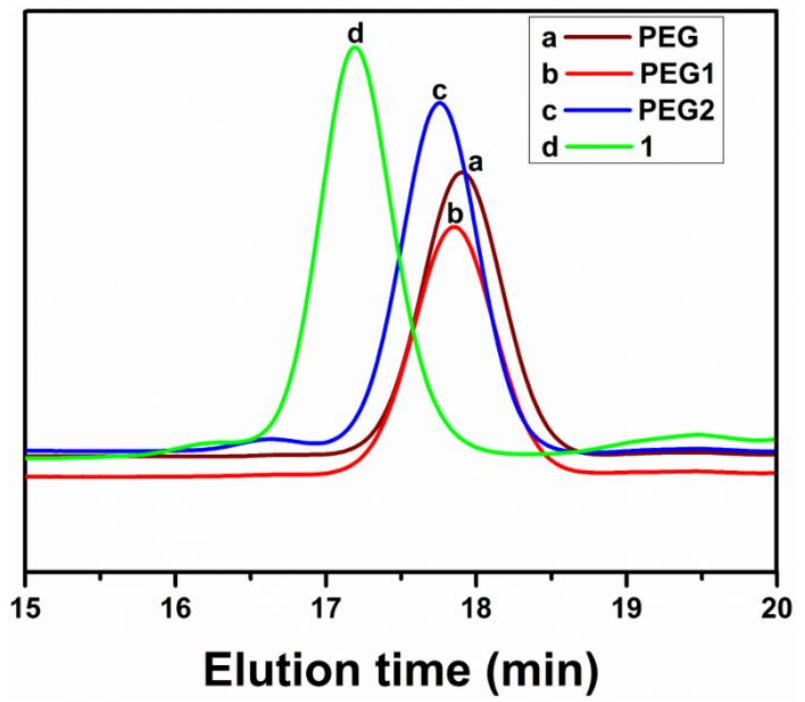

Fig. S1 GPC profiles of (a) PEG, (b) PEG1, (c) PEG2 and (d) 1 (Cb-NA-PEG2000-NA-Cb). See details in Table S1.
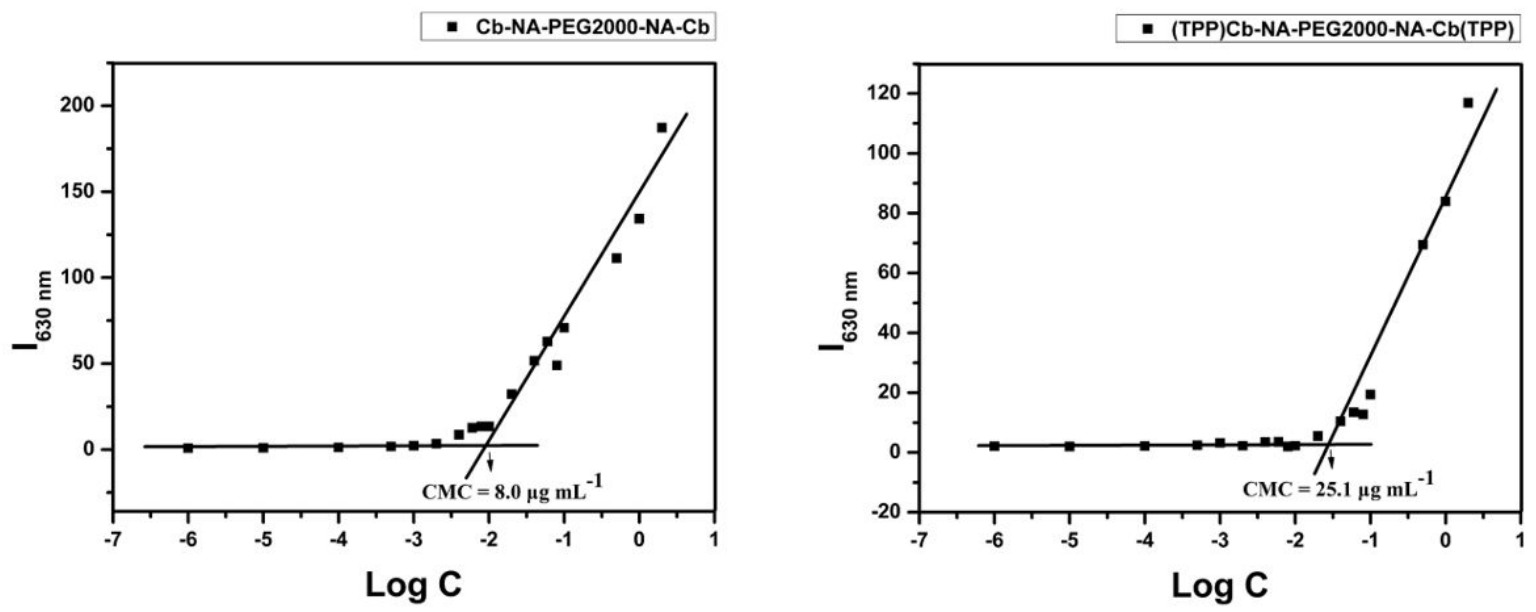

Fig. S2 Critical micelle concentration (CMC) determination of prodrugs: Fluorescence intensity at $\lambda_{\mathrm{em}}=630 \mathrm{~nm}$ of Nile red as function of logarithm of the polymer concentrations in pure $\mathbf{H}_{2} \mathbf{O}$. 

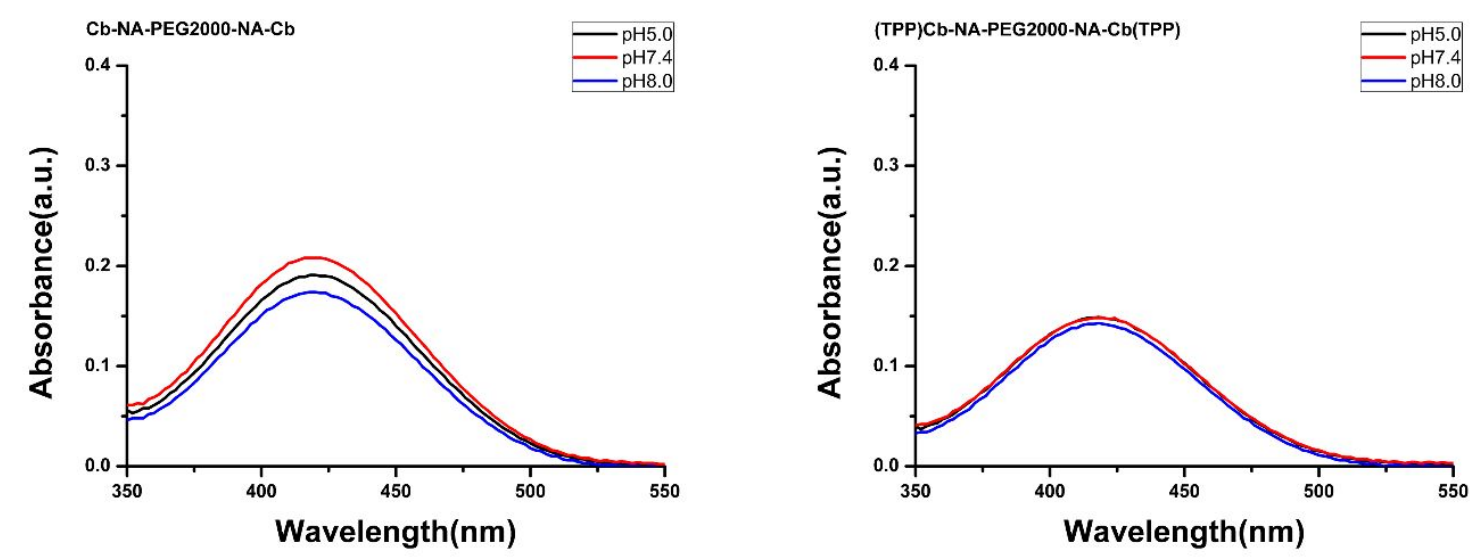

Fig. S3 Same $\lambda \max$ of UV-Vis absorption spectrum also reflected the formation of micelles. UV-vis absorption spectra of prodrugs in PBS buffer solution $(10 \mathrm{mM}){ }^{2}$
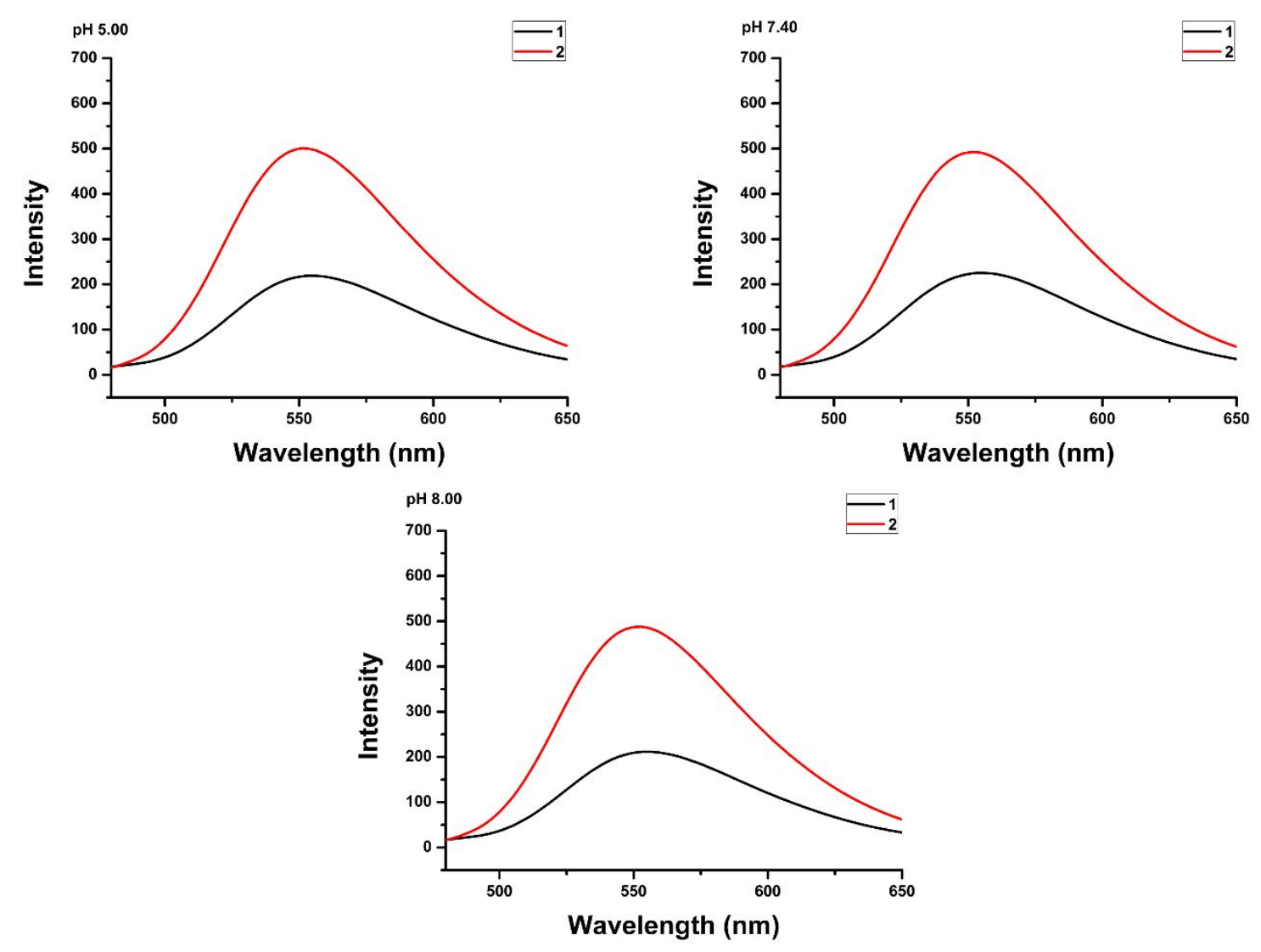

Fig. S4 Fluorescence spectra of (TPP) Cb-NA-PEG2000-NA-Cb(TPP) showed strongest emission in PBS buffer solution (10 $\mathrm{mM}, \mathrm{pH}=5.00,7.40,8.00)$ due to the formation of micelles. 1 and $\mathbf{2}$ represent Cb-NA-PEG2000-NA-Cb and (TPP) Cb-NAPEG2000-NA-Cb(TPP). 

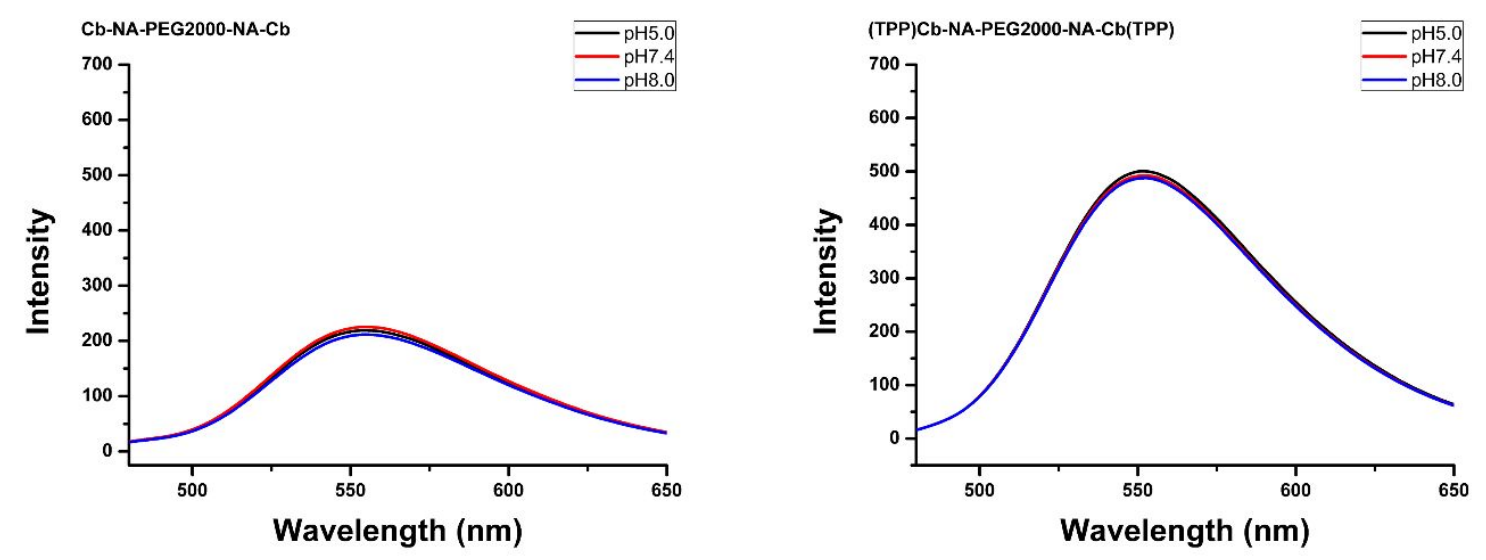

Fig. S5 Fluorescence spectra of prodrugs in PBS buffer solution $(10 \mathrm{mM}, \mathrm{pH}=5.00,7.40,8.00)$ is immune to $\mathrm{pH}$ changes.
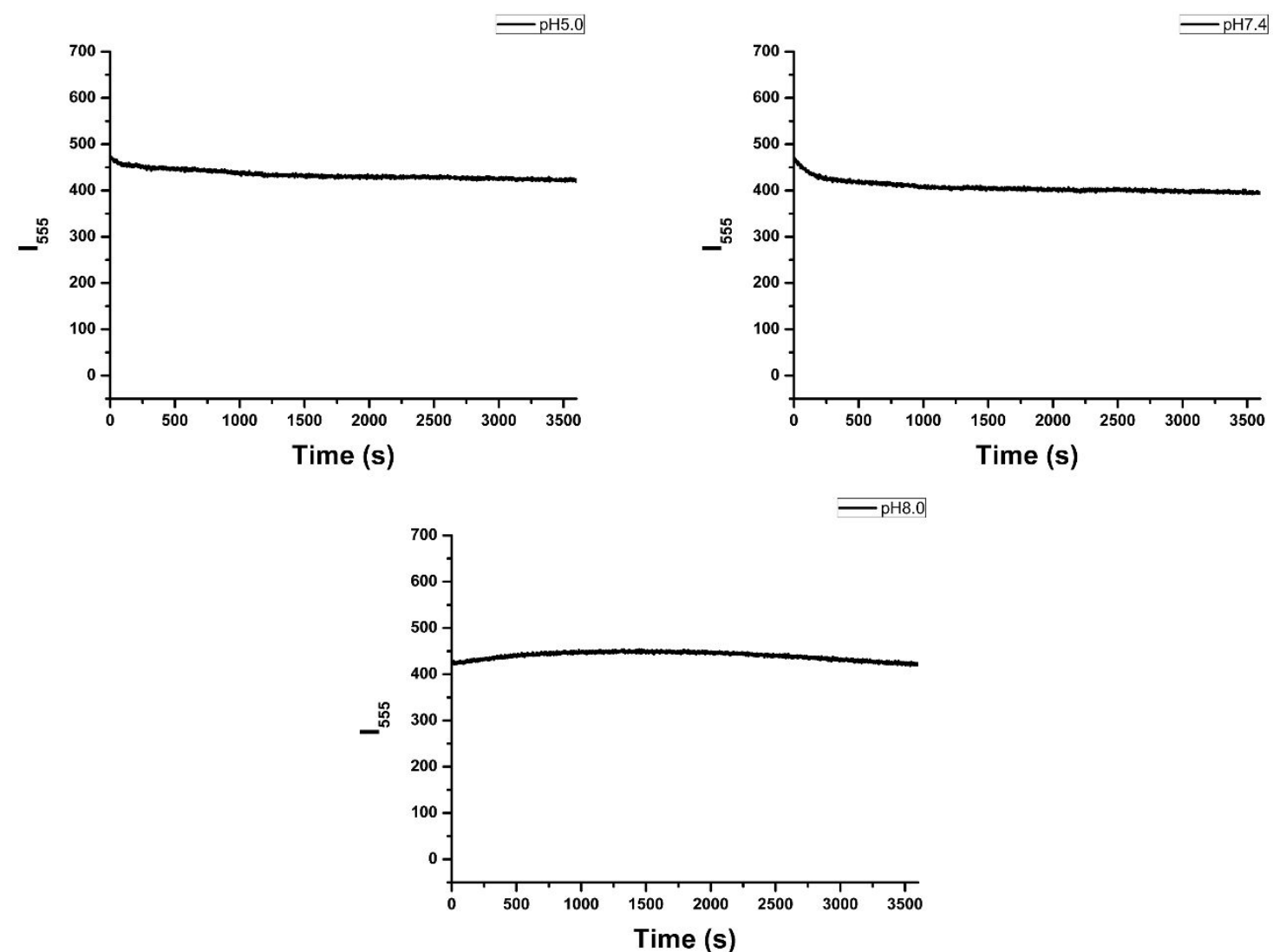

Fig. S6 Temporal profile of (TPP) Cb-NA-PEG2000-NA-Cb(TPP) at $\lambda_{555 \mathrm{~nm}}$ in PBS buffer solution at different pH values (10 $\mathrm{mM}, \mathrm{pH}=5.00,7.40,8.00)$. 

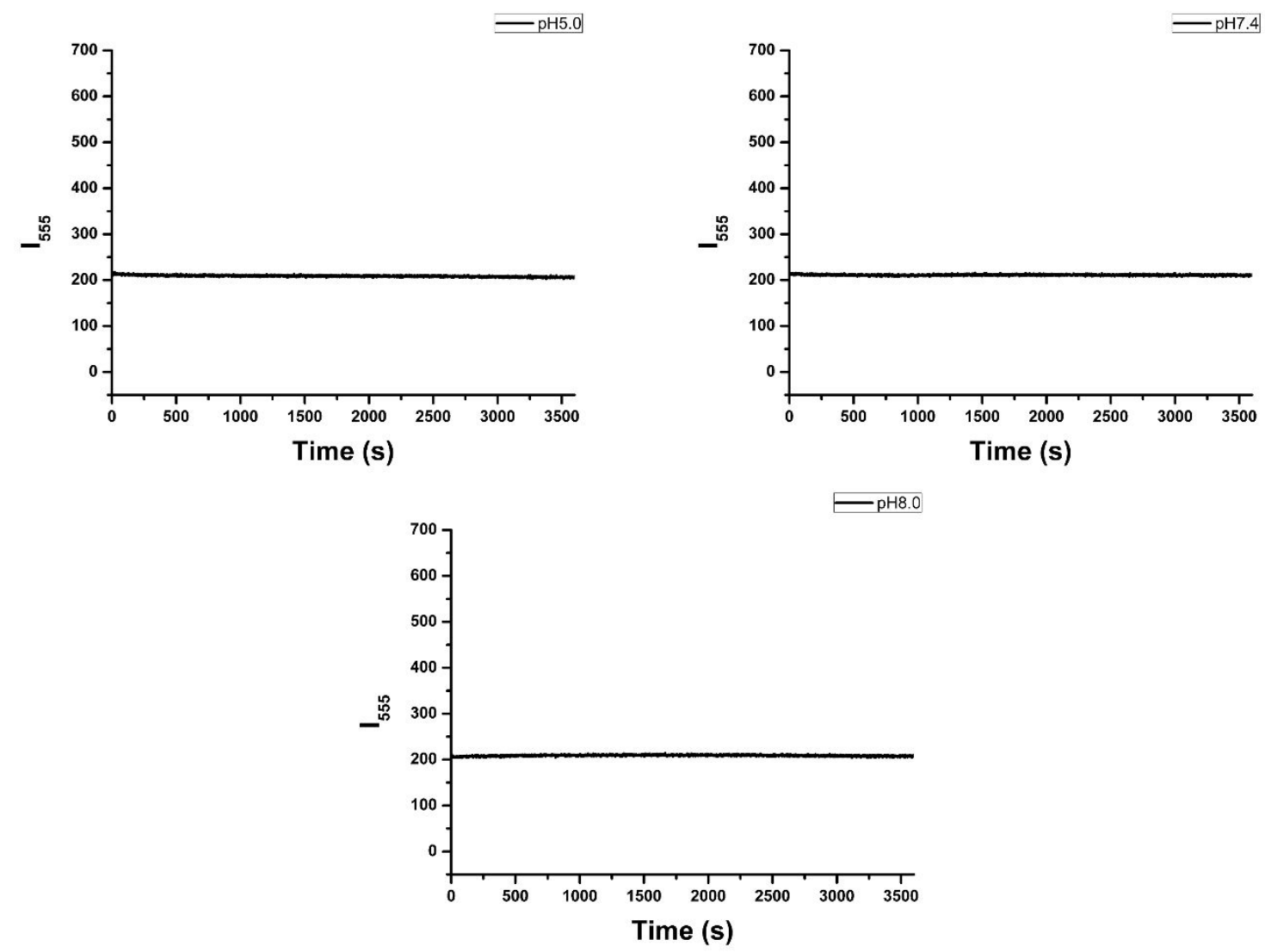

Fig. $\mathbf{S} 7$ Temporal profile of Cb-NA-PEG2000-NA-Cb at $\lambda_{555 \mathrm{~nm}}$ in $\mathbf{P B S}$ buffer solution at different $\mathrm{pH}$ values $(10 \mathrm{mM}, \mathrm{pH}=$ $5.00,7.40,8.00)$.

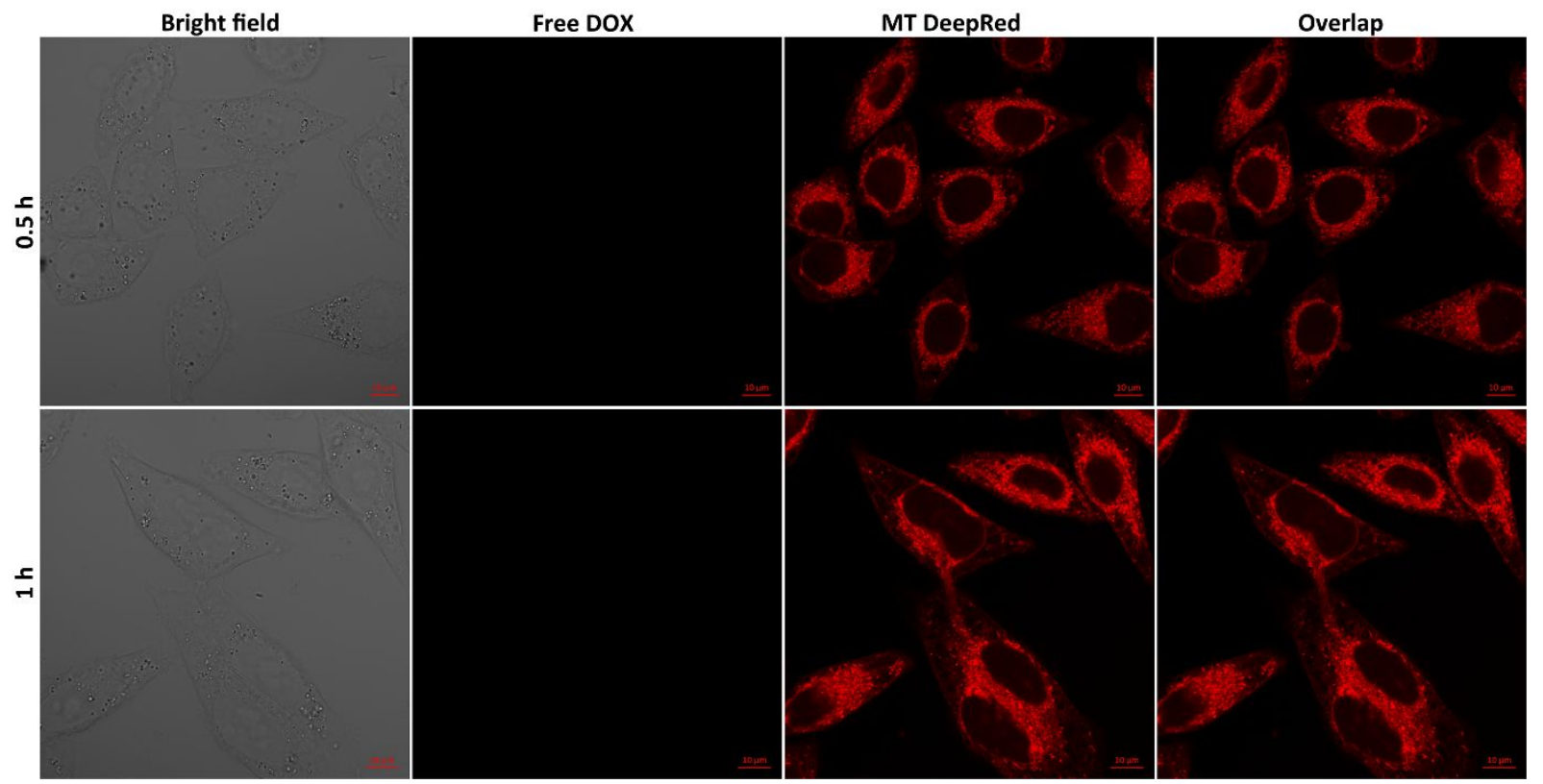

Fig. S8 Cell imaging and mitochondrial targeting of free DOX $(50 \mu \mathrm{g} \mathrm{mL}-1)$ with commercially available mitochondrial dye, Mito Tracker Deep Red FM (MT DeepRed) in HeLa cells for different incubation time ( $0.5 \mathrm{~h}$ and $1 \mathrm{~h})$. 


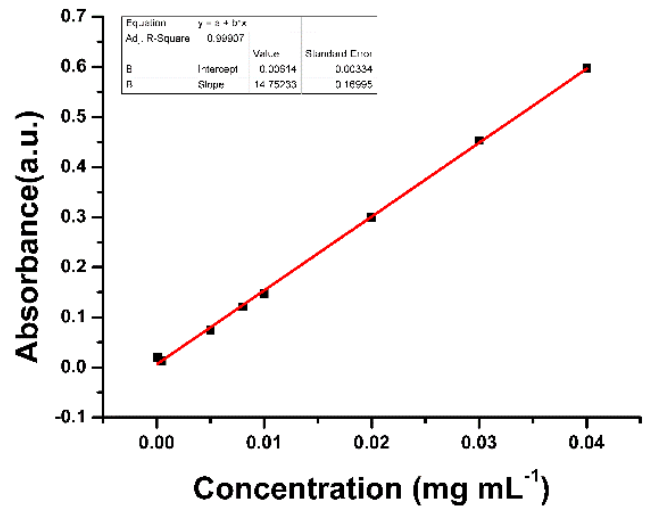

Fig. S9 The free drug standard curve in DMF for DOX encapsulation efficiency (EE) and loading efficiency (LE).
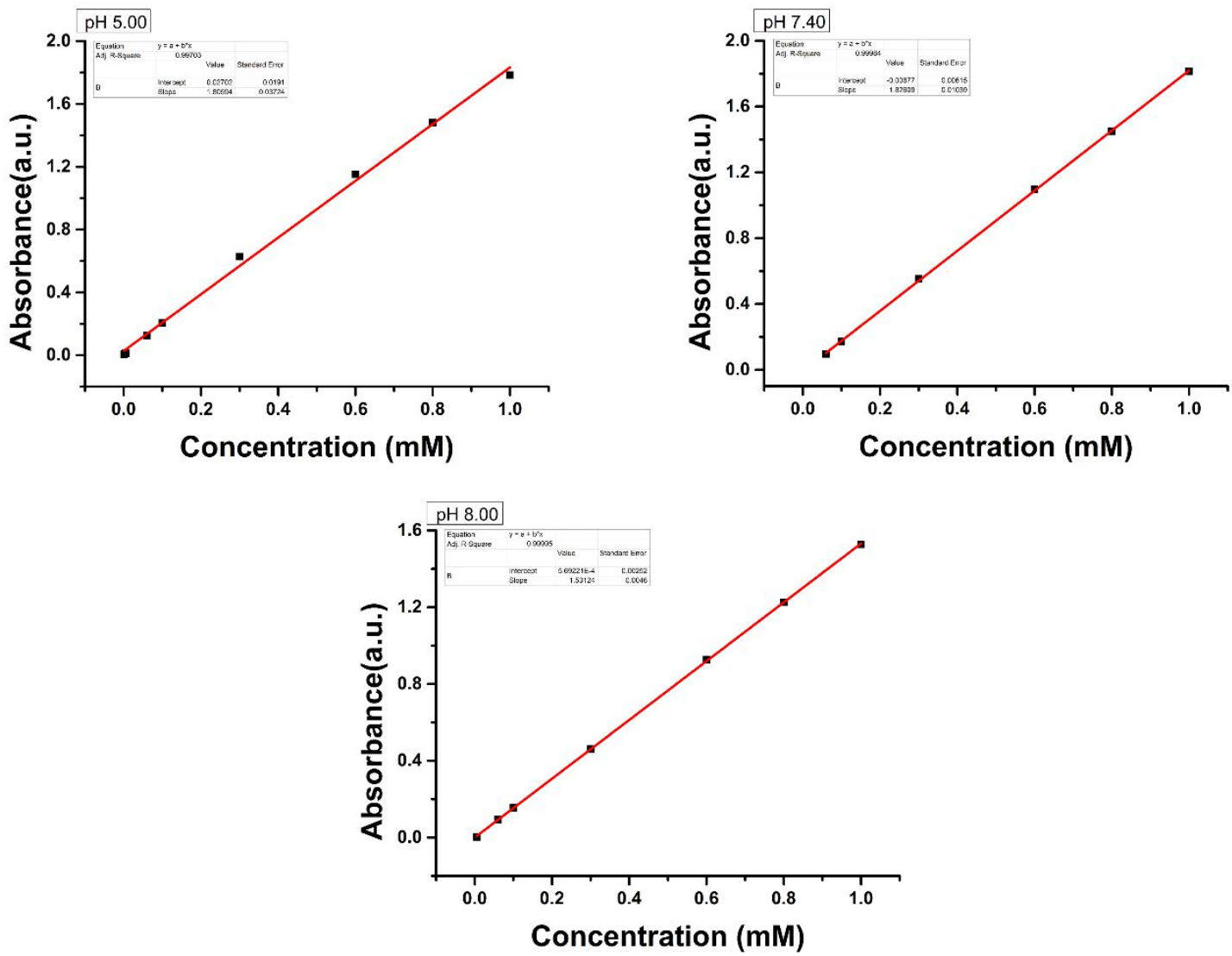

Fig. S10 The free drug standard curve in PBS buffer (10 mM, 0.5\% Tuwen-80) for Cb release. 

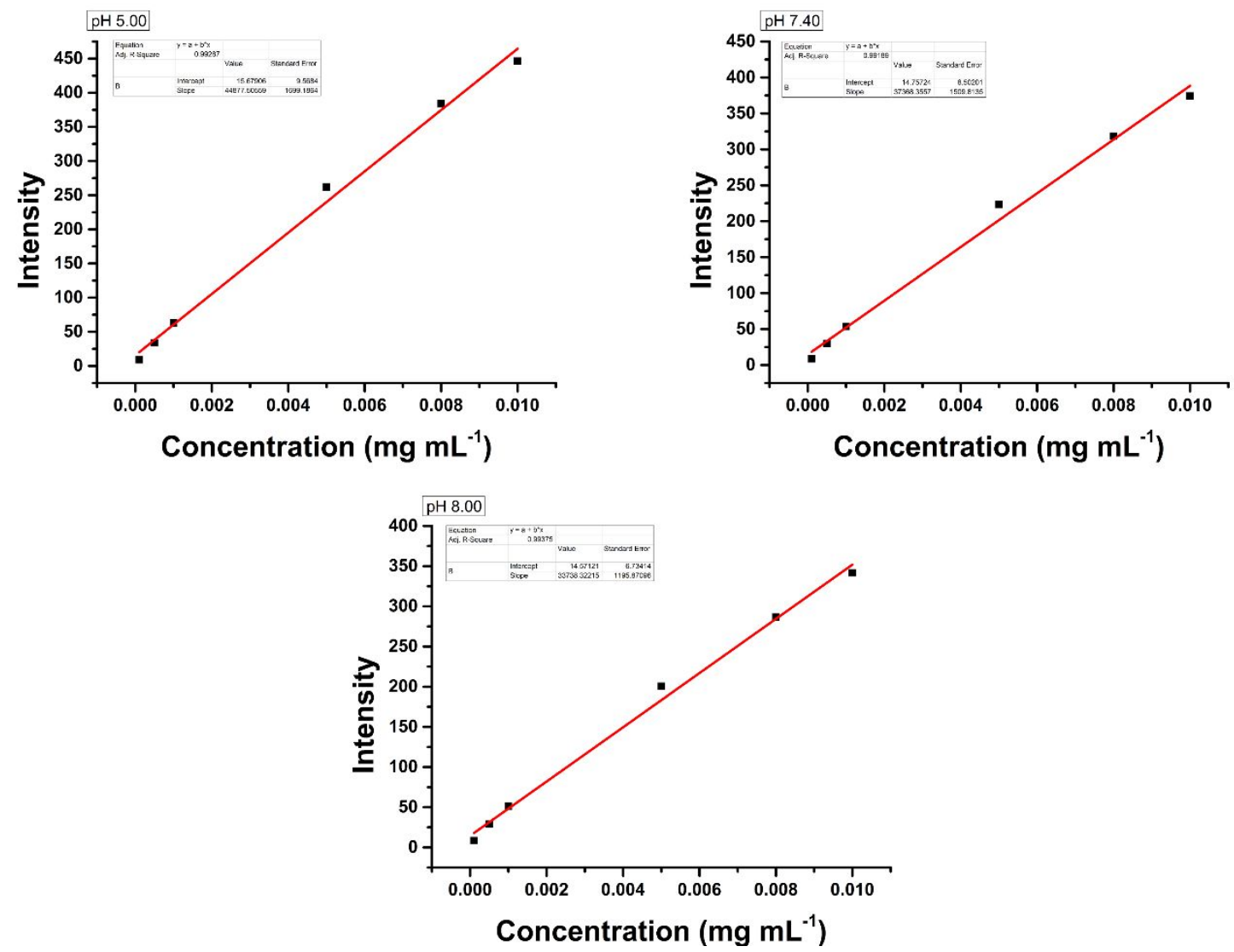

Fig. S11 The free drug standard curve in PBS buffer (10 mM, 0.5\% Tuwen-80) for DOX release.

Table S1 Basic characterisation of the fluorescent polymeric prodrugs

\begin{tabular}{|c|c|c|c|c|c|c|c|}
\hline Sample & $\mathbf{M n}^{a}$ & $\mathbf{M w} / \mathbf{M n}{ }^{a}$ & $\begin{array}{c}\mathrm{CMC}^{b} \\
\left(\mu \mathrm{g} \mathrm{m \mathbf {L } ^ { - 1 }}\right)\end{array}$ & $\begin{array}{l}\text { Size }^{c} \\
(\mathbf{n m})\end{array}$ & $\mathbf{P d I} \mathbf{I}^{c}$ & $\begin{array}{c}\text { Zeta Potential }^{c} \\
(\mathrm{mV})\end{array}$ & $\begin{array}{c}\text { Drug loading } \\
\text { (\%) }\end{array}$ \\
\hline PEG & 1950 & 1.04 & & & & & \\
\hline PEG1 & 2020 & 1.03 & & & & & \\
\hline PEG2 & 2150 & 1.03 & & & & & \\
\hline Cb-NA-PEG2000-NA-Cb & 3140 & 1.04 & 8.0 & $16.2 \pm 0.3$ & $0.504 \pm 0.010$ & $5.43 \pm 0.20$ & $17.6^{d}$ \\
\hline (TPP)Cb-NA-PEG2000-NA-Cb(TPP) & & & 25.1 & $135.9 \pm 9.4$ & $0.311 \pm 0.095$ & $35.10 \pm 2.92$ & $14.1^{d}$ \\
\hline $\begin{array}{c}\text { DOX@(TPP)Cb-NA-PEG2000-NA- } \\
\text { Cb(TPP) }\end{array}$ & & & & $71.5 \pm 1.9$ & $0.293 \pm 0.001$ & $32.37 \pm 5.44$ & $\begin{array}{l}\text { EE: } 95.4 \pm 5.4^{e} \\
\text { LE: } 6.2 \pm 2.9^{e}\end{array}$ \\
\hline
\end{tabular}

${ }^{a}$ Determined by GPC against a poly(ethylene glycol) standard. ${ }^{b}$ Determined by fluorescence analysis using Nile Red as a fluorescence probe. ${ }^{c}$ The size, PdI (size polydispersity index) and zeta potential of the micelles were determined by DLS, $\mathrm{n}=3 .{ }^{d}$ Calculated by the weight fraction of Cb moiety. And all relative calculations were based on theoretical value of molecular weights of prodrugs. ${ }^{e}$ Calculated via the $\mathbf{U V}$-vis analysis, average values. 
Table S2 $\mathrm{IC}_{50}(\mu \mathrm{M})$ values of prodrug systems and free $\mathbf{C b}$ in cancer cells and normal cells along with sensitivity and selectivity ratios and synergistic effect.

\begin{tabular}{|c|c|c|c|c|c|c|c|c|c|c|}
\hline \multirow{2}{*}{ Sample } & \multicolumn{5}{|c|}{ Cancer cells } & \multicolumn{5}{|c|}{ Selectivity ratio $^{a}$} \\
\hline & HEK293(A) & HeLa(B) & HEPG2(C) & A549(D) & MDA MB 231(E) & $\mathbf{A}$ & B & C & D & $\mathbf{E}$ \\
\hline $\mathbf{C b}$ & $591.20 \pm 3.28$ & $325.05 \pm 3.77$ & Low cytotoxicity & Low cytotoxicity & Low cytotoxicity & 0.12 & 0.23 & & & \\
\hline 1 & $457.31 \pm 3.27$ & $691.65 \pm 2.25$ & $602.31 \pm 0.88$ & $560.34 \pm 4.07$ & $288.09 \pm 0.87$ & 0.49 & 0.32 & 0.37 & 0.40 & 0.77 \\
\hline Sensitivity ratio ${ }^{b}$ & 1.3 & 0.47 & & & & & & & & \\
\hline 2 & $114.45 \pm 1.16$ & $28.45 \pm 1.03$ & $34.75 \pm 2.25$ & $32.02 \pm 0.23$ & $44.56 \pm 0.61$ & 2.5 & 9.9 & 8.1 & 8.8 & 6.4 \\
\hline Sensitivity ratio $^{b}$ & 5.2 & $1.1 \times 10^{1}$ & & & & & & & & \\
\hline 3 & $53.84 \pm 0.55$ & $3.20 \pm 0.10$ & $29.17 \pm 0.26$ & $10.74 \pm 0.87$ & $0.069 \pm 0.006$ & 5.0 & 84 & 9.3 & 25 & $3.9 \times 10^{3}$ \\
\hline Sensitivity ratio ${ }^{b}$ & $1.1 \times 10^{1}$ & $1.0 \times 10^{2}$ & & & & & & & & \\
\hline Synergy effect ${ }^{c}$ & 2.1 & 8.9 & 1.2 & 3.0 & $6.5 \times 10^{2}$ & & & & & \\
\hline
\end{tabular}

\section{NMR of various compounds}

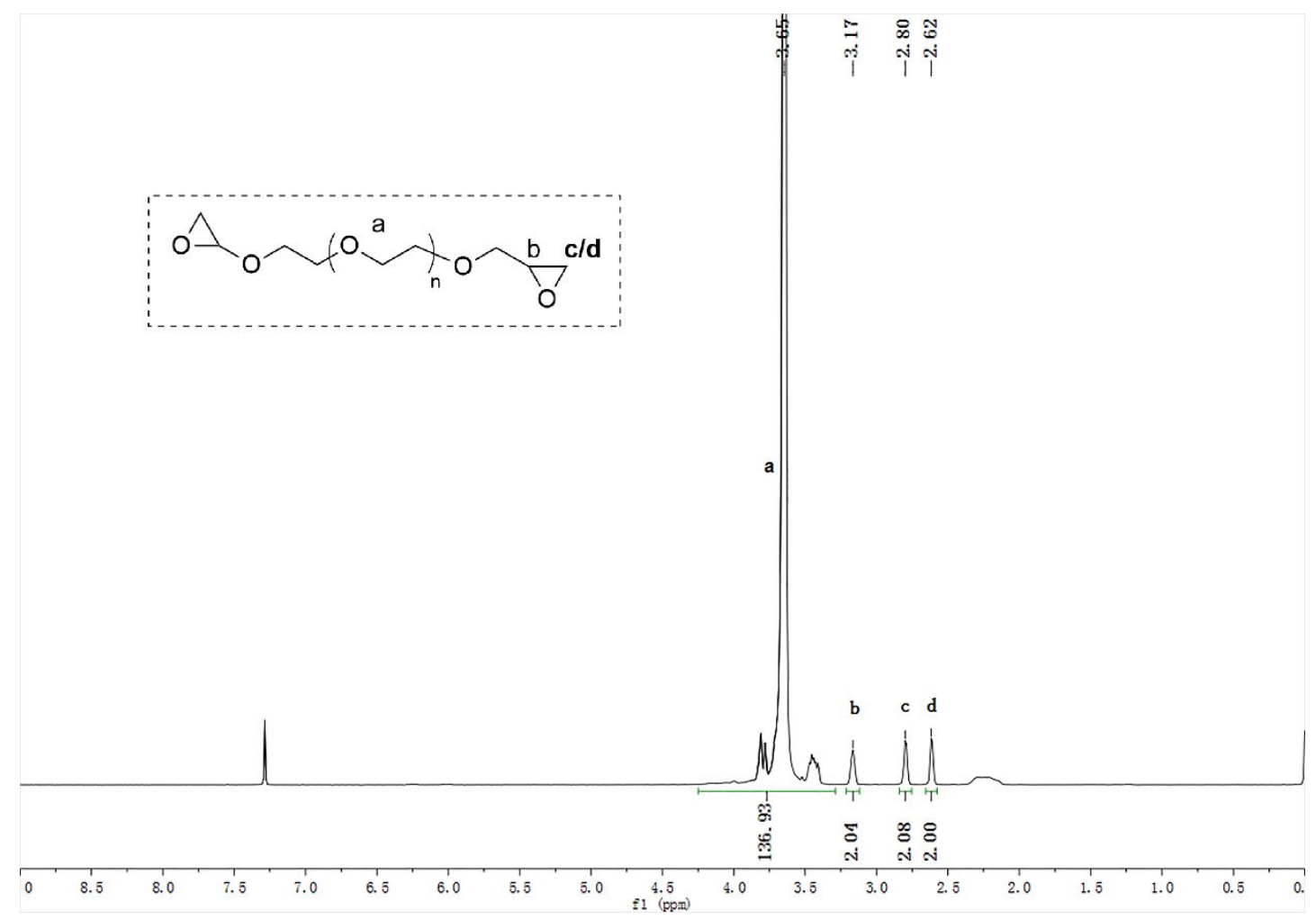

Fig. S12 ${ }^{1} \mathrm{H}$ NMR spectrum of PEG1 in $\mathrm{CDCl}_{3}$. 


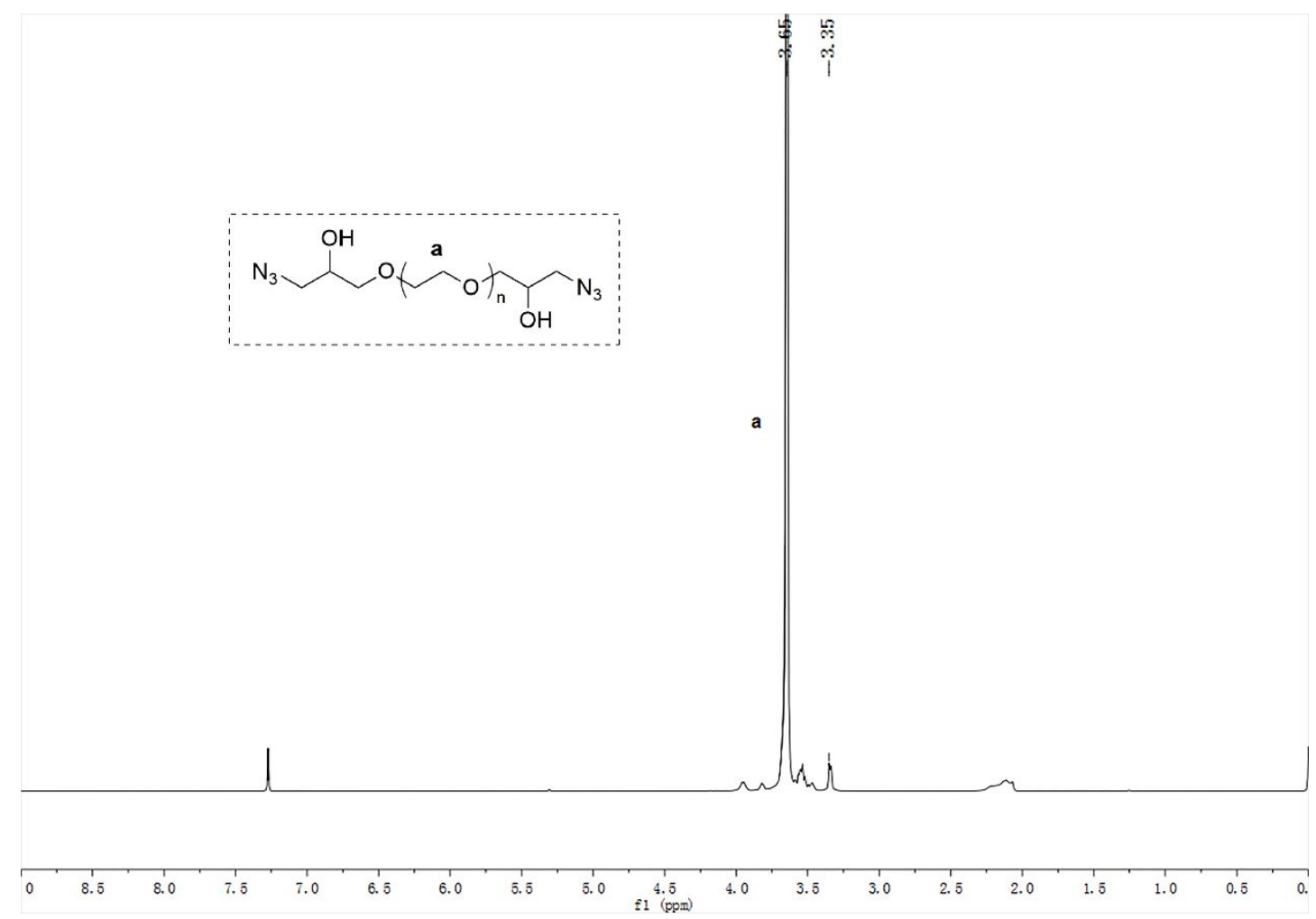

Fig. $\mathbf{S 1 3}{ }^{1} \mathrm{H}$ NMR spectrum of $\mathbf{P E G} 2$ in $\mathrm{CDCl}_{3}$.

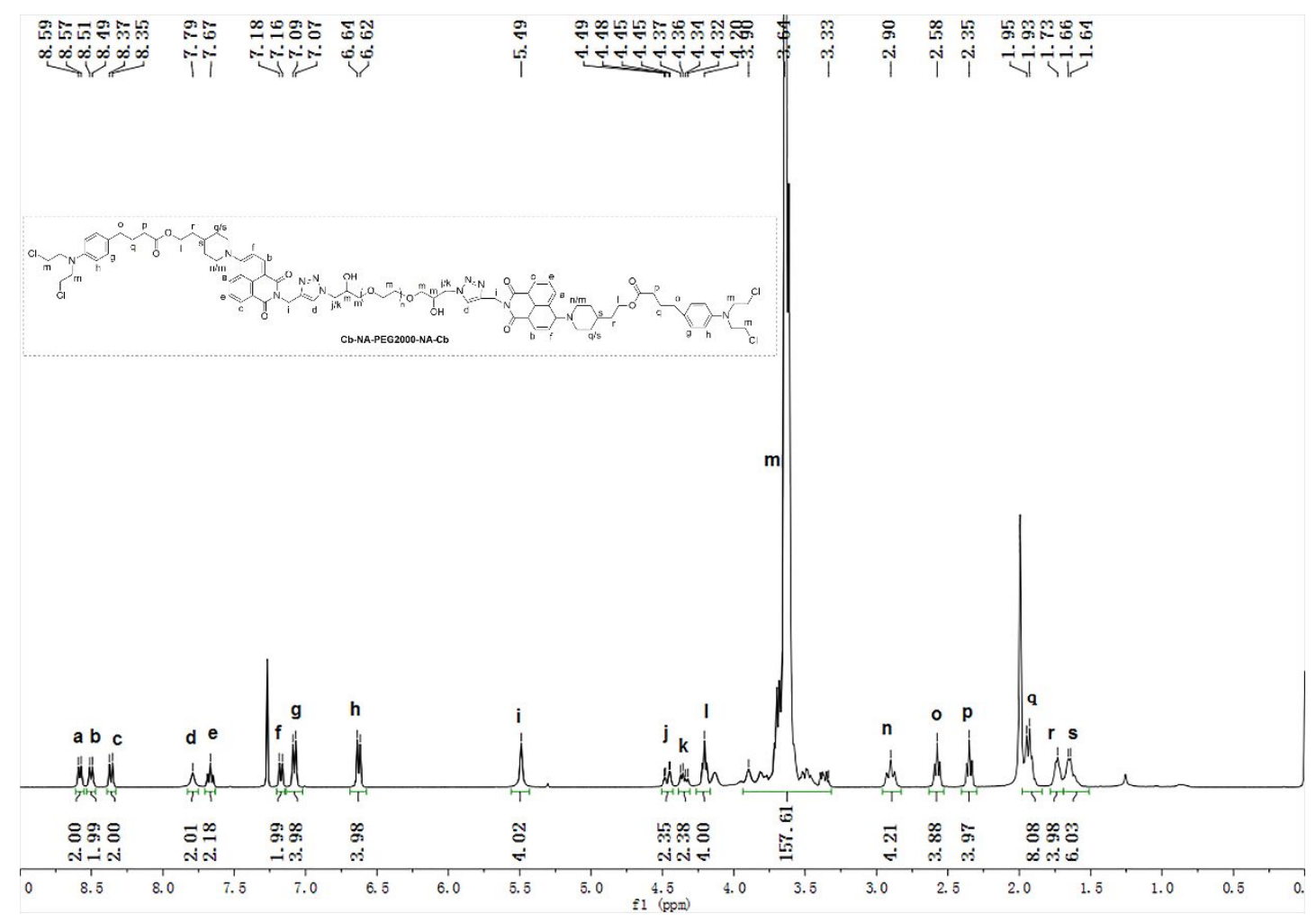

Fig. S14 ${ }^{1} \mathrm{H}$ NMR spectrum of $\mathrm{Cb}-\mathrm{NA}-\mathbf{P E G 2 0 0 0 - N A - C b}$ in $\mathrm{CDCl}_{3}$. 


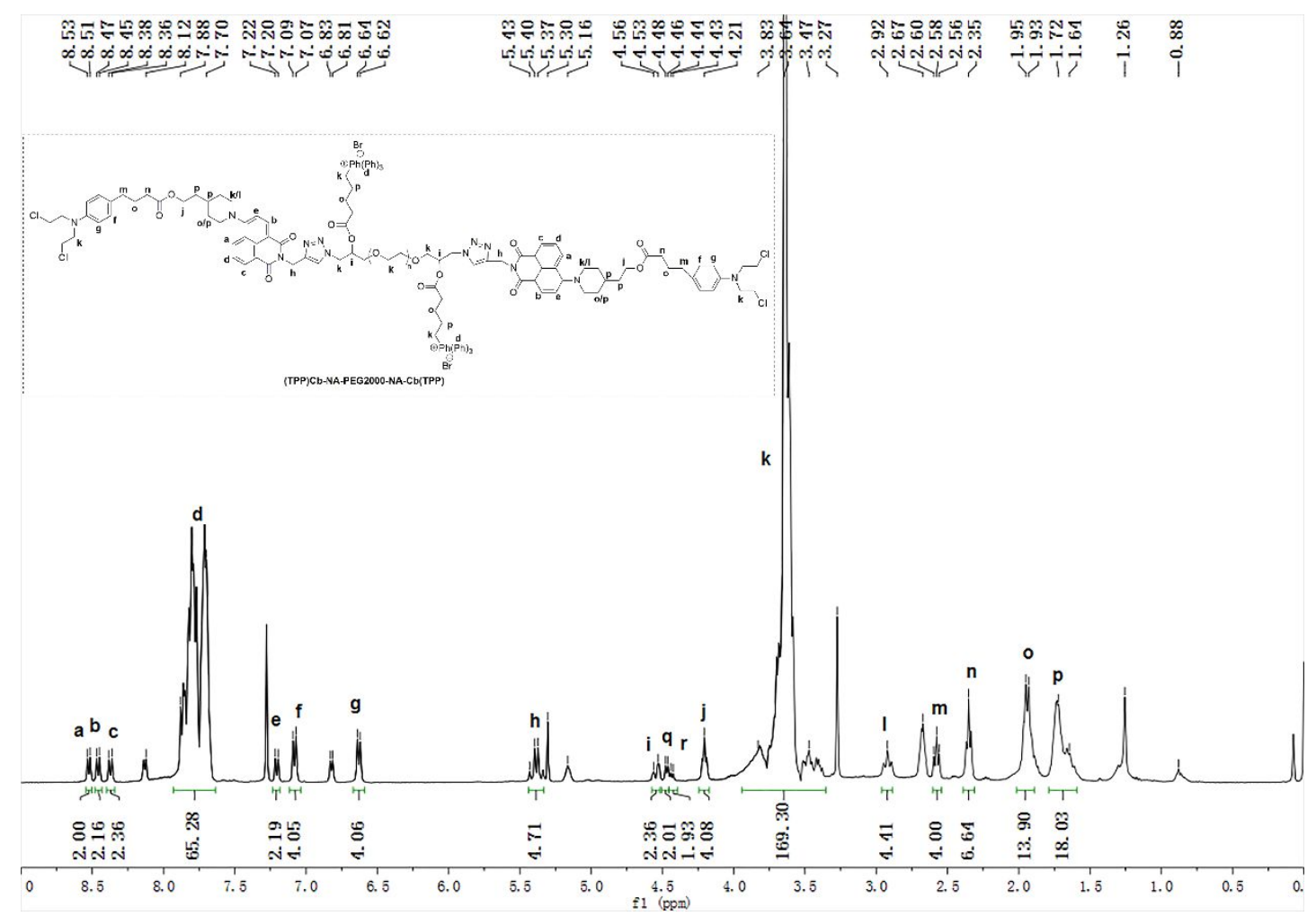

Fig. S15 ${ }^{1} \mathrm{H}$ NMR spectrum of (TPP)Cb-NA-PEG2000-NA-Cb(TPP) in $\mathrm{CDCl}_{3}$.

\section{Reference:}

1. B.-Y. Liu, W.-X. Wu, N. Wang, X.-Q. Yu, Novel biocompatible fluorescent polymeric micelles based on 1,8naphthalimide derivatives for cell imaging, Polym. Chem., 2015, 6, 364.

2. B.-Y. Liu, W.-X. Wu, Y.-H. Liu, C.-J. Jia, X.-L. Yang, J. Li, N. Wang, X.-Q. Yu, Water-soluble mitochondria-targeting polymeric prodrug micelles for fluorescence monitoring and high intracellular anticancer efficiency, Polym. Chem., 2017, $8,5982$.

3. W.-X. Wu, X.-L. Yang, B.-Y. Liu, Q.-F. Deng, M.-M. Xun, N. Wang, X.-Q. Yu, Lipase-catalyzed synthesis of oxidationresponsive poly(ethylene glycol)- $b$-poly( $\beta$-thioether ester) amphiphilic block copolymers, $R S C A d v$., 2016, 6, 11870

4. X. Hu, J. Hu, J. Tian, Z. Ge, G. Zhang, K. Luo, S. Liu, Polyprodurg Amphiphiles: Hierarchical Assemblies for ShapeRegulated Cellular Internalization, J. Am. Chem. Soc., 2013, 135, 17617.

5. Y. -P. Xiao, J. Zhang, Y. -H. Liu, Z. Huang, B. Wang Y. -M. Zhang, X. Yu, Cross-linker polymers with fluorinated bridges for efficient gene delivery, J. Mater. Chem. B, 2017, 5, 8542 .

6. S. -L. Pan, K. Li, L. -L. Li, M.- Yang Li, L. Shi, Y. -H. Liu, X. -Q. Yu, A reaction-based ratiometric fluorescent sensor for the detection of $\mathrm{Hg}$ ( II ) ions in both cells and bacteria, Chem. Commun., 2018, 54, 4955. 